\section{REVISTA CIENTÍFICA RURAL}

ISSN: $1413-8263 \quad 2525-6912$
Revista

Técnico-Científica
$\mathbb{Q}$

EDIUACAMP

urcomp

\title{
EFICIÊNCIA PRODUTIVA DE VACAS LEITEIRAS PRIMÍPARAS E MULTÍPARAS- UMA REVISÃO
}

Patrícia Pinto da Rosa1, Luiza Padilha Nunes², Rodrigo Garavaglia Chesini ${ }^{3}$, Tierri Nunes Pozada ${ }^{4}$, Gabriel Freitas Silva ${ }^{3}$, Juliana da Silva Camacho ${ }^{5}$, Matheus Ramos Faria², Gilliany Nessy Mota ${ }^{2}$, Pablo Tavares Costa ${ }^{1}$

\begin{abstract}
${ }^{1}$ Doutoranda do Programa de Pós-Graduação em Zootecnia (UFPEL), Universidade Federal de Pelotas -RS ptc.agostini@gmail.com, ${ }^{2}$ Mestranda do Programa de Pós-Graduação em Zootecnia, Universidade Federal de Pelotas, ${ }^{3}$ Aluno de graduação em zootecnia- Universidade Federal de Pelotas, ${ }^{4}$ Mestrando Programa de Pós-Graduação em Sistemas de Produção Agrícola Familiar da Faculdade de Agronomia Eliseu Maciel-UFPEL, ${ }^{5}$ Bacharel em Zootecnia pela Universidade Federal de Pelotas
\end{abstract}

RESUMO: A baixa produtividade de leite, altos custos de produção e poucos investimentos em tecnologias, são algumas das dificuldades enfrentadas pelo setor leiteiro. A produção de leite varia com a idade da vaca e o número de partos, sendo que vacas de primeira cria e lactação(primíparas) ainda estão em fase de crescimento corporal e desenvolvimento da glândula mamária e, portanto, teriam menor capacidade produtiva quando comparadas com vacas de duas ou mais crias (multíparas). A formação de lotes é ferramenta essencial dentro da propriedade leiteira, pois vacas primíparas conseguem expressar melhor sua eficiência produtiva quando separadas de multíparas. As multíparas apresentam pico de lactação e produção de leite maior, mas com menor persistência de lactação, quando comparadas com primíparas. A utilização da curva de lactação de primíparas e multíparas, auxilia o produtor a identificar quedas na produção de leite, fazer ajustes na dieta dos lotes, identificando animais superiores, melhorando o desempenho de produção leiteira da propriedade, minimizando os custos. objetivo deste trabalho foi relatar, através de uma revisão bibliográfica, a eficiência produtiva de vacas leiteiras de primeira cria (primíparas) e de mais de uma cria (multíparas) em sistemas de produção de leite.

Palavras-chave: curva de lactação, dieta, lotes, produtividade

\section{PRODUCTIVE EFFICIENCY OF PRIMIPAROUS AND MULTIPAROUS DAIRY COWS}

ABSTRACT: The low dairy yield, high production costs and few investments in technologies are some challenges that need to be faced by the dairy sector. Milk production varies according to animal's age as like the number of calving, and firstlactation (primiparous) cows are still in the phase of body growth and development of 
mammary gland and, therefore, would have lower productive capacity when compared to cows of two or more calves (multiparous). Batch formation, as groups of contemporaries, is an essential tool within dairy farming, since primiparous cows can better express their productive efficiency when shared of from multiparous. Multiparous has a peak lactation and higher milk yield, but less persistence of lactation, when compared to primiparous. The use of the primiparous and multiparous lactation curve helps the producer to identify falls in milk production, to make adjustments in the batch diet, identifying superior animals, improving the dairy production performance and minimizing costs. The objective of this work was to report the productive efficiency of dairy cows (primiparous) and more than one (multiparous) dairy cows in milk production systems.

Keywords: batch, feeding, lactation curve, produtivity

\section{INTRODUÇÃO}

A eficiência produtiva de um rebanho leiteiro depende da interação de uma série de fatores do sistema de produção, onde o entendimento dos mesmos pode auxiliar no manejo nutricional do rebanho.

A baixa produtividade de leite, altos custos de produção e poucos investimentos em tecnologias, são algumas das dificuldades enfrentadas pelo setor leiteiro, deixando o país distante de se tornar um dos principais exportadores de produtos lácteos do mundo (SOUZA et al., 2010). É fundamental que haja oferta de leite com qualidade e quantidade suficiente para suprir a demanda do mercado, e para que haja aumento na produtividade leiteira é necessário atender alguns fatores, como os genéticos, sanitários, ambientais e nutricionais (SILVA et al., 2009).

A atividade leiteira é afetada por fatores intrínsecos ao animal, sabe-se que a produção de leite varia com a idade da vaca e o número de partos (ZANELA, 2015). Esta produção cresce desde a primeira lactação até a vaca atingir sua maturidade fisiológica, mantendo um platô, para depois decrescer suavemente à medida que o animal vai envelhecendo, esse efeito está diretamente relacionado com a ordem de lactação (RANGEL et al., 2009).

Segundo Santos; Fonseca, (2006), vacas de primeira cria e lactação (primíparas) ainda estão em fase de crescimento corporal e desenvolvimento da glândula mamária e, portanto, teriam menor capacidade produtiva. Por outro lado, vacas mais velhas, de segunda ou mais crias (multíparas) tem uma produtividade mais elevada. Neste contexto, objetivo deste trabalho foi relatar a eficiência produtiva de 
vacas leiteiras de primeira cria (primíparas) e de mais de uma cria (multíparas) em sistemas de produção de leite.

Separação de Lotes

De acordo com NRC (2001) vacas primíparas, costumam ter um consumo menor de alimentos e de maneira diferente que vacas multíparas. Além deste fator, vacas primíparas, geralmente são mais temerosas e se encontram em posição social inferior no rebanho, sendo então recomendado sempre que possível a separação de lotes para melhor eficiência do rebanho na propriedade leiteira.

Em estudos de Philips e Rind (2001), onde foram avaliados os efeitos na produção de leite e no comportamento de animais misturando ou não grupos de vacas multíparas e primíparas, que eram mantidos em sistemas rotacionados de pastejo, os autores concluíram que ao misturar vacas primíparas e multíparas, há interferência no tempo de pastejo e no comportamento social do grupo, resultando em queda da produção de leite.

Vacas leiteiras tipicamente são agrupadas em lotes de 40 a 100 animais, onde tem mais facilidade de locomoção e reconhecimento dos mesmos dentro do grupo. Grant; Albright, (2000), afirmam que alguns fatores tendem a interferir no tamanho do lote formado, como espaço de cocho, onde gera competição por água e alimento. Outro fator não menos importante são as interações sociais que existem no rebanho, que dependem do tamanho do espaço disponível para os animais circularem, que irá refletir no consumo de matéria seca e consequentemente na produção de leite dos lotes. O momento da ordenha deve ditar o tamanho máximo do lote, onde uma vaca não deve esperar mais que 1hora e 45 minutos para ser ordenhada, levando em consideração 2 ordenhas diárias (BACH, 2006).

Fazer o uso da ordem de parto é critério adicional de agrupamento de lotes, onde a formação de grupos de animais de primeira lactação e de vacas nas primeiras semanas da lactação é uma prática bastante eficaz. A prática de separação de vacas primíparas de vacas multíparas também é recomendável, pois as primíparas ocupam as posições mais inferiores na ordem de dominância social do rebanho, o que pode resultar em desvantagem competitiva por espaço de cocho e camas com vacas mais 
velhas. A separação de primíparas de multíparas (Tabela 1) pode ser adotada desde o período de transição, imediatamente antes do parto.

Tabela 1. Desempenho de vacas primíparas quando abrigadas juntas ou separadas de vacas multíparas.

Table 1. Performance of primiparous cows when sheltered together or separated from multiparous cows.

\begin{tabular}{lcc}
\hline & Primíparas com multíparas & Primíparas separadas \\
\hline Tempo de alimentação, min/dia & 184 min/dia & 205 min/dia \\
Refeições por dia & 5 & 6 \\
Consumo de concentrado kg/d & $10,1 \mathrm{~kg} / \mathrm{d}$ & $11,6 \mathrm{~kg} / \mathrm{d}$ \\
Consumo de silagem kg/MS/dia & 7,7 & 8,6 \\
Tempo deitadas min/dia & 424 & 461 \\
Períodos de repouso/dia & 5,3 & 6,3 \\
Produção de leite, $\mathrm{kg}$ em 130 dias & 2388 & 2595 \\
Gordura do leite $\%$ & 3,92 & 3,97 \\
\hline
\end{tabular}

Grant; Albright, (2001).

Primeira ordenha

Para as novilhas primíparas, o período do pós-parto é de muita sensibilidade, pois existem inúmeros fatores desconhecidos e novos para o animal, onde acaba o colocando em situação de estresse e desconforto (SCHÜLLER, 2014). As mudanças na rotina destas novilhas, essencialmente em relação ao ambiente, ao manejo pelos funcionários no momento da ordenha, necessitam de estratégias para tornar essa ação mais tranquila para que o animal tenha uma resposta positiva na hora que entrar na sala de ordenha (PHELPS, 1992). As estratégias de manejo durante o préparto visam tornar essa habituação mais normal possível, pois um problema existente, é o fato de a primípara ser muito reativa, de ocasionar até mesmo acidente de trabalho por conta dessa reatividade, pode ser minimizada quando é feito certo manejo repetidamente (LINN, 2004).

Um manejo se torna eficiente quando as novilhas são conduzidas uma vez ao dia por funcionários até a sala de ordenha (onde serão ordenhadas futuramente), para passar por um processo de condicionamento (LUCCI, 1993). Momentos antes de entrarem para a ordenha, as novilhas ficam na sala de espera, onde já passam por um refrescamento, manejo muito importante para vacas no pré-parto. Tal forma de manipulação, além de condicionar os animais, afeta positivamente em seu bem-estar, 
resultando na melhoria de sua produção futura de leite e da qualidade de seu colostro, bem como no peso da futura cria (KEYSERLINGK et al., 2012).

Em um segundo momento, conforme os estudos de Lucci (1993), as novilhas devem entrar na sala de ordenha, de forma calma e tranquila sem a presença de barulhos ou ruídos estranhos, onde são condicionadas a pararem na linha de ordenha e recebem um pequeno estímulo, e logo em seguida são liberadas, repetindo-se este processo por 21 dias antes do parto. Estes processos garantem um animal mais tranquilo no pós-parto, já acostumado a sala de ordenha, evitando possíveis acidentes e não se faz necessário com vacas multíparas onde já estão condicionadas a rotina de ordenha (WATHES, et al., 2007).

\section{Manejo Nutricional}

A inconstância da exigência nutricional entre períodos de lactação, é uma questão problemática no momento da alimentação de rebanhos leiteiros, onde este acontecimento se dá pela forma da curva de lactação e a variação que a mesma apresenta entre primíparas e multíparas (INGVARTSEN et al., 2013). As variações ocorrem quanto à taxa de aumento na produção de leite, que se dá desde o parto até o pico de produção, ao valor da produção máxima, ao intervalo em dias do parto ao alcance da produção máxima e persistência da produção ao longo da lactação (CAMERON et al. 1998).

Segundo Vuuren e Chilibroste, (2013), animais de elevada produção onde a fase de lactação é alta, requerem um grande consumo de alimento, pois a produção de leite é o maior determinante da exigência nutricional, já que é proporcionalmente maior que a demanda para mantença, ganho de peso e gestação. No período pósparto, a ingestão de matéria seca é baixa, mas a exigência nutricional do animal é superior, e nesta fase se faz necessário dietas com altos valores nutricionais para suprir tais demandas e garantir um bom funcionamento metabólico a vaca lactante, (WATHES, et al., 2007). O aumento do consumo de matéria seca pelo animal se da com o avançar do período de lactação onde ocorre queda da demanda nutricional para produção de leite, onde dietas de alta densidade nutricional têm elevado teor de concentrados, enquanto dietas de baixa densidade são formuladas com alto teor de forragens, e apresentam normalmente custos mais baixos por kg de matéria seca (SANTOS et al., 1998). 
O manejo nutricional do rebanho leiteiro é planejado conforme a exigência de cada propriedade, de cada lote formado e se possível de cada animal, onde formular uma dieta para cada animal é inviável na prática, e muitas propriedades adotam uma dieta única para todos os animais em lactação, ou dividindo em lotes por produção e aumentando apenas o volume de concentrado (CASTRO et al., 2008). Nestes rebanhos, tanto vacas em início quanto aquelas em final de lactação são animais com alta exigência nutricional, que acaba propiciando a formulação de uma dieta de alta densidade nutricional para todos os animais, tendo como parâmetro a exigência das vacas de maior produção (CAMERON et al. 1998).

Segundo Olson (2002), o uso de uma única dieta ocasiona à superalimentação e ganho excessivo de condição corporal por vacas menos produtivas, outro resultante desta prática é o maior uso de alimentos concentrados por litro de leite produzido, o que aumenta o custo alimentar. Quanto mais grupos forem formados, mais a dieta fica próxima da exigência nutricional de cada animal do rebanho, onde em situações de baixo preço de leite e alta dispersão na produção por animal dentro do rebanho, agrupamentos bem feitos podem ser uma maneira efetiva de reduzir o custo de alimentos concentrados por litro de leite produzido (RENAUDEAU et al., 2012). Agrupar bem não é uma opção tecnológica para ser enfatizada apenas em rebanhos de alta genética e onde se visa alta produção por vaca, muito pelo contrário, esta prática tende a ser mais eficaz financeiramente em rebanhos menos produtivos (SANTOS et al., 2001).

Produção de Leite

Vacas primíparas quando são agrupadas separadamente das multíparas e são manejadas adequadamente tendo uma dieta de transição bem formulada, é quase inexistente animais com baixa produção, nos primeiros 3 meses do pós-parto (WATHES, et al., 2007). Estes lotes, a produção diária de vacas primíparas nos 60 dias iniciais ao pós-parto deve ser representada por $70 \%$ da produção média deste grupo. Isso significa que, em um rebanho com produção média diária no grupo de vacas em primeira lactação de $35 \mathrm{~kg} / \mathrm{dia}$, a produção diária no teste mensal dos animais nos primeiros 60 dias em lactação deve ser de pelo menos 24 a $25 \mathrm{~kg} / \mathrm{dia}$ (SANTOS et al., 2006). O pico de produção de leite está diretamente relacionado com a produção total durante a lactação, estima-se que para cada quilograma a mais de 
leite no pico de lactação, a vaca irá produzir cerca de 150 a $300 \mathrm{~kg}$ a mais de leite durante a lactação completa. No entanto, essas respostas são distintas de acordo com o grupo de animais (DRACKLEY, 1999).

Novilhas de primeira lactação apresentam uma maior persistência de produção que aqueles em sua segunda ou em lactação superior, onde define-se persistência como 1 menos a perda relativa de produção mensal após o animal ter atingido seu pico de produção. Normalmente a persistência de produção de primíparas varia entre 92 e $96 \%$, e de multíparas entre 88 e 94\%. Isso significa que o impacto do aumento de $1 \mathrm{~kg}$ de leite no pico de produção nas vacas de primeira lactação é maior do que aquele nas vacas com mais de uma lactação (SANTOS et al., 2001). Após o parto (11a a $20^{\text {a }}$ semana) se estabiliza o peso vivo, reduzindo a produção de leite e a ingestão de alimentos atinge o ponto máximo onde o animal consegue ingerir todos os nutrientes que necessita para mantença e produção, recuperando, desta forma, as reservas adiposas mobilizadas no início da lactação (LUCCI, 1993).

\section{Curvas de Lactação}

As curvas de lactação de uma vaca leiteira são representadas por variações gráficas da produção diária de leite em função do período de duração da lactação sendo utilizada para medir a produção de leite durante toda a lactação (GONÇALVES et al., 2002). O conhecimento do desempenho das curvas de lactação, propicia no uso correto de manejo alimentar, na seleção e no descarte de animais conforme um padrão preestabelecido da capacidade de produção (RENNÓ et al., 2006). Na figura 1 do texto mostra um exemplo de curva de lactação para produção de leite. 
Multípara-classe1 $\Delta$ Multípara-classe2 $\bullet$ Primípara-classe1 $*$ Primípara-classe2

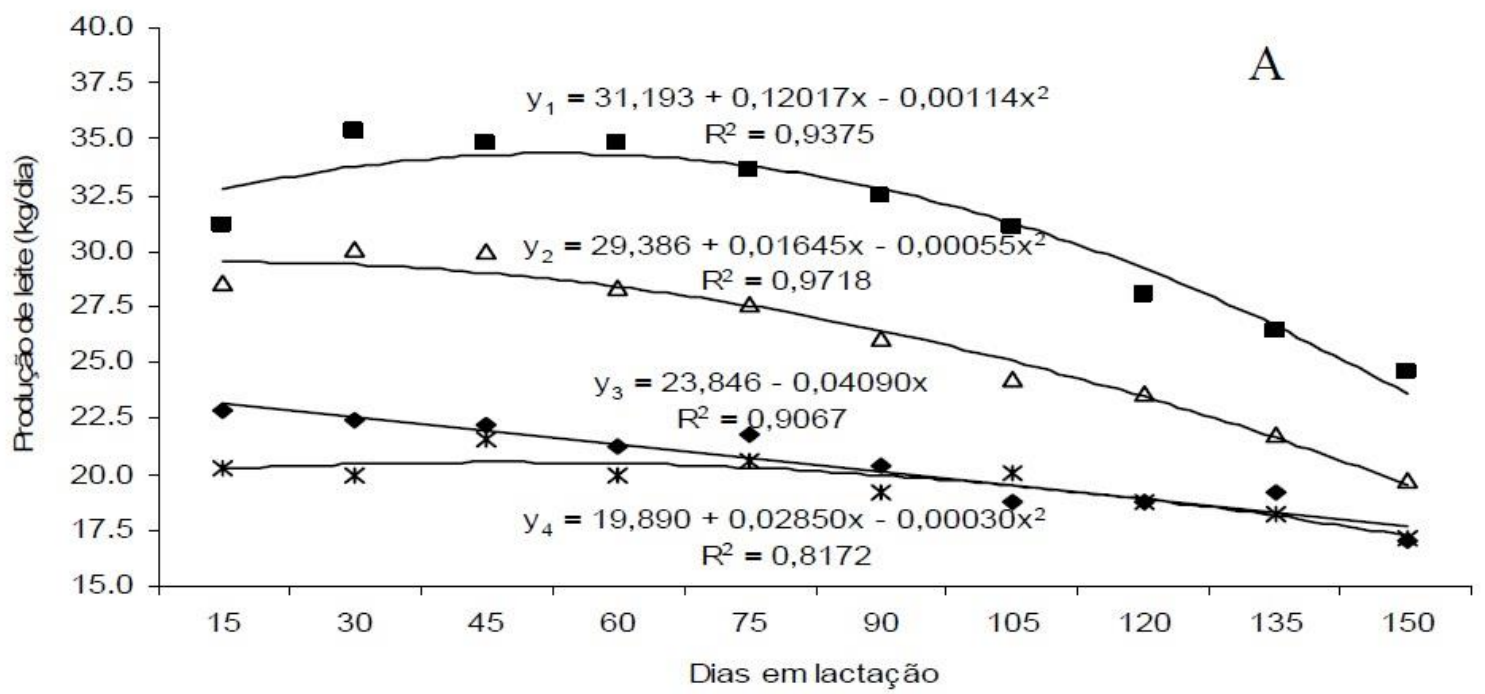

- Multípara - classe1 $\Delta$ Multípara - classe2 • Primípara-classe1 * Primípara-classe2

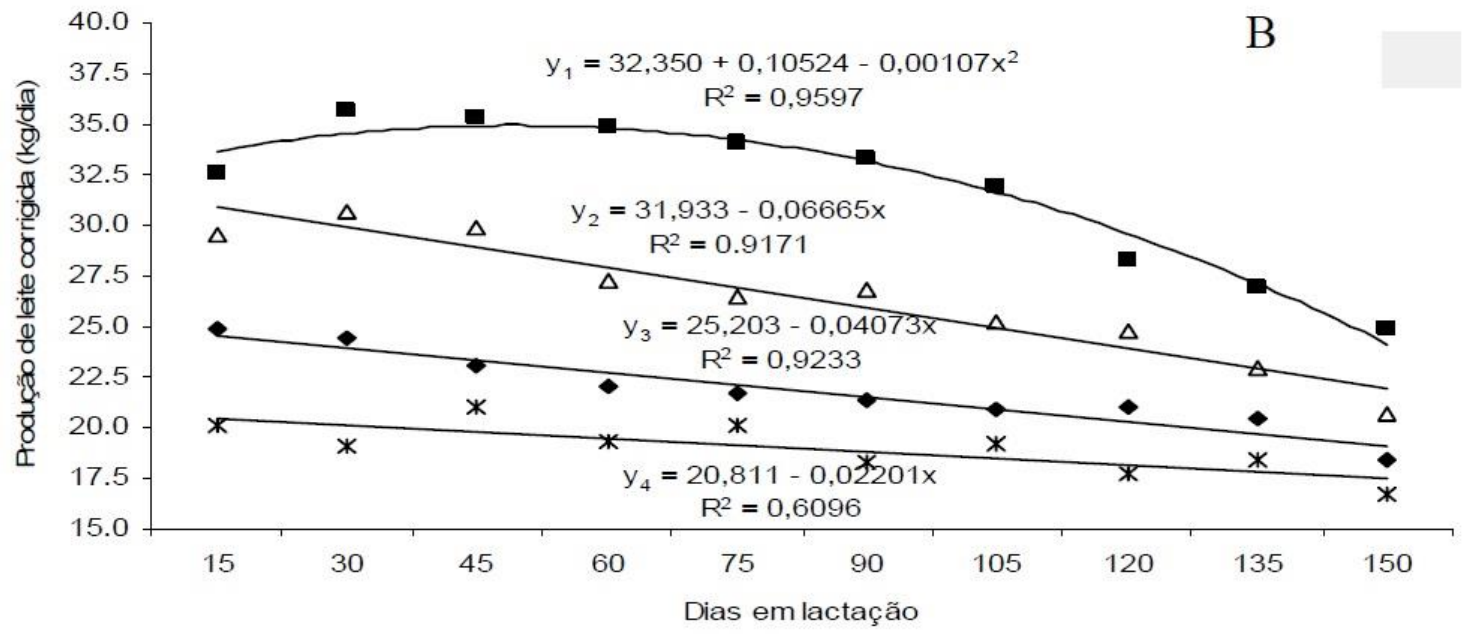

Figura 1- Curva de lactação da produção de leite $(\mathrm{kg} / \mathrm{dia})(\mathrm{A})$ e produção de leite corrigida para 3,5\% de gordura (kg/dia) (B) até os 150 dias de lactação, segundo a condição corporal ao parto, para primíparas e multíparas. (RENNÓ et al., 2006).

Figure 1 - Lactation curve of milk production ( $\mathrm{kg} /$ day) (A) and milk production corrected to $3.5 \%$ fat $(\mathrm{kg} /$ day) (B) up to 150 days of lactation, according to body condition at for primiparous and multiparous women. (RENNÓ et al., 2006).

O uso de comparações das formas das curvas de lactações entre grupos distintos de animais, com composições raciais diferentes, idade ao parto, conformações de rebanhos distintos é importante pois podem ser obtidas informações 
sobre a eficiência desses grupos propiciando um melhor controle da produção (GROENEWALD; VILJOEN, 2003). Curvas de lactação apresentam fase crescente (35 dias após parto), fase de pico que é representada pela produção máxima observada, sendo a terceira fase de declínio contínuo até chegar ao final da lactação GRILLO et al., 2015).

Uma vaca apresenta lactação mais persistente, comparada a outra com produção equivalente, se possuir pico mais baixo e, por conseguinte, um formato da curva de lactação mais achatado, onde este fato resulta na distribuição mais equilibrada da produção de leite no decorrer da lactação (GENGLER, 1996). A persistência na lactação está diretamente interligada a aspectos econômicos da atividade leiteira, pois sua melhoria pode contribuir para a redução de custos no sistema de produção (TEKERLI et al., 2000; JAKOBSEN et al., 2002).

Dekkers et al. (1998) ao estudarem as variáveis econômicas que estavam relacionadas com a persistência de lactação, notaram que o valor econômico é influenciado pelos gastos com alimentação, reprodução e sanidade da vaca de leite, sendo então o retorno econômico adquirido pela produção extra de leite, devido a persistência da lactação destes animais.

Animais com curvas de lactação mais persistentes, tem uma demanda energética mais constante em toda lactação, podendo ser utilizados alimentos mais baratos, ou seja, vacas que apresentam uma menor curva de inclinação, podem fazer uso de dietas de menor custo do que aquelas com produção diária mais elevada durante a fase inicial da lactação (GONÇALVES et al., 2002). Curvas de lactação mais planas, indicam reduzido estresse fisiológico, devido à redução ou ausência de produções elevadas no pico de lactação, o que acaba diminuindo a incidência de problemas reprodutivos e de doenças de origem metabólica, ocasionando quedas de custos no sistema de produção na propriedade leiteira (GROSSMAN et al., 1999; TEKERLI et al., 2000).

Um outro fator importante que compõe a curva, é o pico de lactação, sendo definido como a produção máxima alcançada durante toda a lactação. Em um estudo com vacas Holandesas, Gonçalves et al. (2002), observaram que o pico ocorreu aos 38 dias pós-parto. Existem variações do pico de lactação dentro da raça Holandês, pois Cobuci et al. (2004) relataram que este pico ocorreu entre 60 e 90 dias de lactação. Já em vacas zebuínas ou mestiças, o pico de lactação, se estabeleceu no primeiro dia, ou seja, iniciando na produção máxima com ausência da fase de 
inclinação do parto ao pico (PAPAJCSIK; BODERO, 1988). O mesmo também foi relatado na raça Gir por Rebouças et al. (2008).

Nem todas as vacas ou lotes tem curvas de lactação iguais, pois além da variação genética, a ação dos fatores que vão determinar sua forma, costuma variar conforme influência de diferentes elementos, como ordem de parição, a idade da vaca e a estação de parição; sendo maior a persistência em vacas primíparas que em vacas multíparas de $3^{a}$ e $4^{a}$ lactação (TEKERLI et al., 2000; COBUCl et al., 2011).

Em um estudo de Tekerli et al. (2000) com vacas Holandesas, na Turquia, apontaram que o pico de lactação e a produção de vacas primíparas foram, respectivamente de, 26,6 kg e $6.220 \mathrm{~kg}$. Para vacas de segunda parição, a produção no pico foi de $30,3 \mathrm{~kg}$, enquanto a produção total foi de $6.693 \mathrm{~kg}$. Para as ordens de parição maiores que dois, os animais produziram $30,5 \mathrm{~kg}$ no pico e $6.710 \mathrm{~kg}$ no final da lactação, demonstrando que animais multíparos possuem maiores produção no pico e produção total de leite quando comparados a animais primíparas.

Nos Estados Unidos, Dematawewa et al. (2007), com lotes de vacas Holandesas, detectaram que animais de primeira ordem de parto com duração da lactação de 305 dias tiveram produção média no pico de lactação de $33,3 \mathrm{~kg}$, ocorrendo aos 94 dias; para os animais de terceira ordem de parição ou maior, também com duração de 305 dias de lactação, a produção no pico foi de $44,3 \mathrm{~kg}$ e ocorreu aos 51 dias.

\section{CONCLUSÕES}

Vacas primíparas conseguem expressar melhor sua eficiência produtiva quando separadas de multíparas, mostrando que a formação de lotes nos rebanhos leiteiros se faz necessário, podendo ser utilizado como uma ferramenta eficaz. As multíparas apresentam pico de lactação e produção maior, mas com menor persistência de lactação, quando comparadas com primíparas.

O conhecimento da curva de lactação de primíparas e multíparas, auxilia o produtor a identificar quedas na produção de leite, fazer ajustes na dieta dos lotes, identificando animais superiores, melhorando o desempenho de produção leiteira da propriedade, minimizando os custos. 


\section{REFERÊNCIAS}

BACH, A.; IGLESIAS, C.; DEVANT, M.; RÀFOLS, N. Performance and feeding behavior of primiparous cows loose housed alone or together with multiparous cows. Journal of Dairy Science, v.89, p.337-342, 2006.

CAMERON, R. E. B.; DYK, P. B., HERDT; T. H. Dry cow diet, management, and energy balance as risk factors for displaced abomasum in high producing dairy herds. Journal of Dairy Science, v.81, p.132-139, 1998.

CASTRO, D.; RIBEIRO, C.; SIMÕES, J. Medicina da produção: estratégias alimentares no pós-parto das vacas leiteiras. Revista eletrônica de veterinária, Aveiro, v. IX, n. 10, 2008.

COBUCI, J. A.; EUCLYDES, R. F.; COSTA, C. N.; LOPES, P. S.; TORRES, R. de A.; PEREIRA, C. S. Analysis of persistency in the lactation of Holstein cows using testday yield and random regression model. Revista Brasileira de Zootecnia, v.33, n.3, p. 546-554, 2004.

COBUCl, J. A.; EUCLYDES, R. F.; TEODOR, R. L.; VERNEQUE, R. da S.; LOPES, P. S.; SILVA, M. de A. Genetic parameters for milk production by using random regression models with different alternatives of fixed regression modeling. Revista Brasileira de Zootecnia, v. 40, n.3, p. 557-56, 2011.

DEKKERS, J. C. M.; TEM HAG, J. H.; WEERSINK, A. Economic aspects of persistency of lactation in dairy cattle. Livestock Production Science, v.53, n.3, p. 237252, 1998.

DEMATAWEWA, C. M. B.; PEARSON, R. E.; VANRADEN, P. M. Modeling extended lactations of holsteins. Journal of Dairy Science, v.90, n.8, p. 3924-3936, 2007.

DRACKLEY, J.K. Biology of dairy cows during the transition period: the final frontier? Journal of Dairy Science, v. 82, p. 2259-2273,1999. 
GRANT, R. J.; ALBRIGHT, J. L. Feeding behaviour. In: D'Mello, J.P.F. Farm Animal Metabolism and Nutrition, ed. CABI Publishing. Wallingford, Oxon, UK, p.365-382, 2000.

GRANT, R. J.; ALBRIGHT, J. L. Effect of Animal Grouping on Feeding Behavior and Intake of Dairy CattleJournal of Dairy Science, 84(E. Suppl.): E156-E163, 2001.

GENGLER, N. Persistency of lactation yields: A review. Interbull Bulletin, v.12, p. 97102, 1996.

GRILLO, F.G., GUIMARÃES, A.L.L.; cOUTO, S.R.B.; FIGUEIREDO, M.A.; PALHANO, H.B. Comparação da taxa de prenhez entre novilhas, primíparas e multíparas da raça Nelore submetidas à inseminação artificial em tempo fixo. Revista Brasileira de Medicina Veterinária, v. 37, n. 3, p. 193-197, 2015.

GONÇALVES, T. M.; OLIVEIRA, A. I. G.; FREITAS, R. T. F.; PEREIRA, I. G. Curvas de lactação em rebanhos Holandesa no estado de Minas Gerais. Escolha do melhor ajuste. Revista da Sociedade Brasileira de Zootecnia, v.31, n.4, p.1689-1694, 2002.

GROENEWALD, P. C. N.; VILJOEN, C. S. Application of Bayesian inference in the comparison of lactation curves of Merino ewes. Journal of Agricultural Biological and Environmental Statistics, v. 8, n.1, p. 75-83, 2003.

GROSSMAN, M.; HARTZ, S. M.; KOOPS, W. P. Persistency of lactation yield: A novel approach. Journal of Dairy Science, v. 82, n.10, p. 2192-2197,1999.

INGVARTSEN, K.L. and MOYES, K. Nutrition, immune function and health of dairy cattle. Animal, v 7, p.112-122, 2013.

KEYSERLINGK, V.; BARRIENTOS, M.A.G.; ITO, A.; GALO, Weary. Benchmarking cow comfort on North American free stall dairies: Lameness, leg injuries, lying time, facility design, and management for high-producing Holstein dairy cows. Journal Dairy Science, v.95 p. 7399-7408,2012. 
JAKOBSEN, J. H.; MADSEN, P.; JENSEN, J.; PEDERSEN, L. G.; CHRISTENSEN, L. G.; SORENSEN, D. A. Genetic parameters for milk production and persistency for Danish Holstein estimated in random regression models using REML. Journal of Dairy Science, v.85, n.6, p. 607-1616, 2002.

LINN, J.; TERRE, M.; CASPER, D.; RAETH-KNIGHT, M. Feed efficiency of lactating dairy cows. p. 38-46 in Proc. 65th Minnesota Nutr. Conf., St. Paul, MN. Univ. Minnesota, St. Paul. 2004.

LUCCI, C. S. Alimentação da vaca leiteira: bases técnicas. IN: PEIXOTO, A. M.; MOURA, J. C.; FARIA, V. D. Bovinocultura leiteira, fundamentos da exploração racional. Piracicaba, FEALQ. v.9, p.135-152, 1993.

NATIONAL RESEARCH COUNCIL. In: 7th Revised Edition of Nutrient Requirements of Dairy Cattle. National Academy Press, Washington, DC, 2001.

OLSON, J. Estratégias de nutrición para vacas en transición. Hoard's Dairyman, v. 88, p. $288,2002$.

PAPAJCSIK, I. A.; BODERO, J. Modeling lactation curves of friesian cow in a subtropical climate. Animal Production, v.47, n.1, p. 201-207, 1988.

PHELPS, A. Vastly superior first lactations when heifers fed separately. Feedstuffs, v.64, p.11-18, 1992.

PHILLIPS, C. J. C; RIND, M. I. The Effects on Production and behaviour of mixing uniparous and multiparous cows. Journal of Dairy Science, v.84, p.2424-2429, 2001.

RANGEL A. H. N.; GUEDES P. L. C.; ALBUQUERQUE, R. P. F.; NOVAIS, L. P.; LIMA JÚNIOR D. M. Desempenho produtivo leiteiro de guzerá. Revista Verde de Agroecologia e Desenvolvimento Sustentável, v. 4, p.85-89, 2009. 
REBOUÇAS, G. F.; GONÇALVES, T. M.; MARTINEZ, M. L.; AZEVEDO, J.; KOOPS, W. Novas funções para estimar a produção de leite em 305 dias de lactação de vacas da raça Gir. Revista Brasileira de Zootecnia, v.37, n.7, p.1222-1229, 2008.

RENAUDEAU, D.; COLLIN, A.; YAHAV, S.; DE BASILIO, V.; GOURDINE, J.L. and COLLIER, R.J. Adaptation to hot climate and strategies to alleviate heat stress in livestock production. Animal, v6, p. 707-728, 2012.

F.P. Rennó, J.C. Pereira, A.D.F. Santos, N.G. Alves, C.A.A. Torres, L.N. Rennó, P.Z. Balbinot. Efeito da condição corporal ao parto sobre a produção e composição do leite, a curva de lactação e a mobilização de reservas corporais em vacas da raça Holandesa. Revista Arquivo Brasileiro de Med. Veterinária e Zootecnia., v.58, n.2, p.220-233, 2006

SANTOS, J. E.; AMSTALDEN, M. Effects of nutrition on bovine reproduction. In: ARQUIVOS DA SOCIEDADE BRASILEIRA DE VETERINÁRIA, 26, 1998, Atibaia. Anais... Atibaia. 1998. p.19-89.

SANTOS, J. E.; SANTOS, F. A. P.; JUCHEM, S. O. Monitoramento do manejo nutricional em rebanhos leiteiros. In: Reunião Anual da Sociedade Brasileira de Zootecnia (38: 2001: Piracicaba). Anais... CD-ROM, p. 1-18.

SANTOS, M. V.; FONSECA, L. F. L. Estratégias para controle de mastite e melhoria da qualidade do leite. Barueri: Manole, 2006. 314p.

SILVA, C. V.; LANA, R. D. P.; CAMPOS, J. D. S.; QUEIROZ, A. D.; LEÃO, M. I.; ABREU, D. D. Consumo, digestibilidade aparente dos nutrientes e desempenho de vacas leiteiras em pastejo com dietas com diversos níveis de concentrado e proteína bruta. Revista Brasileira de Zootecnia, v. 38, n. 7, p. 1372-1380, 2009.

SOUZA, R.; DOS SANTOS, G. T.; VALLOTO, A. A.; DOS SANTOS, A. L.; GASPARINO, E.; DA SILVA, D. C.; SANTOS, W. B. R. Produção e qualidade do leite de vacas da raça Holandesa em função da estação do ano e ordem de parto. Revista Brasileira de Saúde e Produção Animal, v. 11, n. 2, p. 25-37, 2010. 
SCHÜLLER, L.K.; BURFEIND, O. and HEUWIESER,W. Impact of heat stress on conception rate of dairy cows in the moderate climate considering different temperature-humidity index thresholds, periods relative to breeding, and heat load indices. Theriogenology, V 81, p. 1050-1057, 2014.

TEKERLI, M.; AKINCI, Z.; DOGAN, I.; AKCAN, A. Factors affecting the shape of lactation curves of Holstein cows from the Balikesir province of Turkey. Journal Dairy Science, v.83, n.6, p. 1381-1386, 2000.

VUUREN, V.A.M. and CHILIBROSTE, P. Challenges in the nutrition and management of herbivores in the temperate zone. Animal, v. 7, p. 19-28, 2013.

WATHES, D.C.; CHENG, Z.; BOURNE, N.; TAYLOR V.J.; COFFEY, M.P. and BROTHERSTONE, S. Diferences betem primíparas and multíparos dairy cows in the inter-relationships between metabolic traits, milk yield and body condition score in the periparturient period. Domestic Animal Endocrinol, v33, p. 203-225, 2007.

ZANELA, M.B. Leite Instável Não Ácido (LINA): do campo a indústria. In: VI Congresso Brasileiro de Qualidade do Leite, Curitiba. p.1-16, 2015. 